2. Drysdale AT, Grosenick L, Downar J, Dunlop K, Mansouri F, Meng Y, et al. Restingstate connectivity biomarkers define neurophysiological subtypes of depression. Nat Med. 2017;23:28-38.

3. Etkin A, Maron-Katz A, Wu W, Fonzo GA, Huemer J, Vertes PE, et al. Using fMRI connectivity to define a treatment-resistant form of post-traumatic stress disorder. Sci Transl Med. 2019;11:eaal3236.
4. Scott JC, Matt GE, Wrocklage KM, Crnich C, Jordan J, Southwick SM, et al. A quantitative meta-analysis of neurocognitive functioning in posttraumatic stress disorder. Psychol Bull. 2015;141:105-40.

\title{
Linking actions with their consequences within the ventrolateral orbital cortex
}

\author{
Dan C. $\mathrm{Li}^{1}$ and Shannon L. Gourley (iD) ${ }^{1,2}$ \\ Neuropsychopharmacology (2020) 45:227-228; https://doi.org/10.1038/s41386-019-0498-1
}

Humans and rodents can associate actions with their outcomes and modify expectations when associations change. These cognitive adaptions accommodate change and presumably optimize decision-making. For example, we might modify our driving route when construction blocks our path or abstain from alcohol when we need to drive, expecting that both actions will deliver us safely home. Across species, medial prefrontal cortical regions are involved in linking actions with valued outcomes, but contributions of the orbitofrontal cortex remain contentious. One issue is that the orbitofrontal cortex occupies a large territory, yet is sometimes treated as a homologous structure. A related concern is the assumption that ventromedial subregions, like lateral regions, specialize in stimulus-outcome associations (linking cues, rather than actions, with likely outcomes) agnostic to action-outcome associations. Nevertheless, poor or aberrant decision-making is commonplace in neuropsychiatric illnesses, necessitating a full dissection of how action-outcome associations form, update, and solidify.

Parkes et al. [1] made important in-roads in resolving controversies. Rats were trained to associate two actions with a single food reward; then the actions were paired with different, unique rewards in the days preceding a devaluation test. Inactivation of the ventrolateral orbitofrontal cortex (VLO) either during the final training days or during the subsequent probe test blocked the ability of rats to choose actions based on the value of respective rewards. Meanwhile, VLO inactivation had no effect when action-outcome contingencies had not changed during training. In another investigation, inactivation of the VLO immediately following the violation of a familiar action-outcome association in mice occluded optimal responding in a later test, even when the VLO was back "on-line" [2]. Thus, the VLO appears necessary for stabilizing newly formed or updated action-outcome associations, which then guide future behavior.

Notably, linking actions with their outcomes involves dendritic spine plasticity on excitatory neurons in the VLO [2]. Specifically, updating action-outcome expectations reduces thin-type dendritic spines, considered immature, on layer $\mathrm{V}$ neurons. Meanwhile, the proportion of mushroom-shaped spines, considered mature, increases, potentially solidifying newly modified action -outcome associations to optimize future decision-making. Remarkably, inactivating VLO neurons upon the violation of familiar action-outcome associations not only blocks response updating, but also inhibits dendritic spine plasticity in an activitydependent manner [2]. These patterns strongly suggest that dendritic spine plasticity on excitatory VLO neurons is necessary for forming action-outcome associations, consistent with evidence that orbitofrontal neurons are capable of forming and maintaining long-term reward-related memory to support behavioral adaptations [3].

Orbitofrontal neurons display a rich diversity of functionally distinct populations based on input/output patterns, many of which make unique contributions to flexible decision-making $[3,4]$. A key question is thus: What inputs to the VLO help to form/update action-outcome associations? Are these inputs distinct from those supporting other associations, e.g., stimulus - outcome associations? Basolateral amygdala (BLA) projections are one candidate. BLA lesions alter the reward-related coding properties of orbitofrontal neurons [5], and BLA $\rightarrow$ orbitofrontal cortical connections appear necessary for certain forms of reinforcement learning [4], including in primates ([6] and references therein). Whether and how these "bottom-up" connections are involved in forming action-outcome associations should be resolved.

\section{FUNDING AND DISCLOSURE}

DCL and SLG are supported by the National Institute of Mental Health and National Institute on Drug Abuse at the National Institutes of Health (grant numbers 044297, 117103, 117873). The authors declare no competing interests.

\section{ADDITIONAL INFORMATION}

Publisher's note: Springer Nature remains neutral with regard to jurisdictional claims in published maps and institutional affiliations.

\footnotetext{
${ }^{1}$ Graduate Training Program in Neuroscience, Departments of Pediatrics and Psychiatry, Emory University School of Medicine, Yerkes National Primate Research Center, Emory University, 954 Gatewood Road NE, Atlanta, GA 30329, USA and ${ }^{2}$ Graduate Training Program in Molecular and Systems Pharmacology, Emory University, Atlanta, GA 30329, USA Correspondence: Shannon L. Gourley (shannon.l.gourley@emory.edu)
}

Received: 17 July 2019 Revised: 19 August 2019 Accepted: 20 August 2019

Published online: 2 September 2019 


\section{REFERENCES}

1. Parkes SL, Ravassard PM, Cerpa JC, Wolff M, Ferreira G, Coutureau E. Insular and ventrolateral orbitofrontal cortices differentially contribute to goal-directed behavior in rodents. Cereb Cortex. 2018;28:2313-25.

2. Whyte AJ, Kietzman HW, Swanson AM, Butkovich LM, Barbee BR, Bassell GJ, et al. Reward-related expectations trigger dendritic spine plasticity in the mouse ventrolateral orbitofrontal cortex. J Neurosci. 2019;39:4595-605.

3. Namboodiri VMK, Otis JM, van Heeswijk K, Voets ES, Alghorazi RA, RodriguezRomaguera J, et al. Single-cell activity tracking reveals that orbitofrontal neurons acquire and maintain a long-term memory to guide behavioral adaptation. Nat Neurosci. 2019;22:1110-21.
4. Groman SM, Keistler C, Keip AJ, Hammarlund E, DiLeone RJ, Pittenger C, et al. Orbitofrontal circuits control multiple reinforcement-learning processes. Neuron. 2019. https://doi.org/10.1016/j.neuron.2019.05.042.

5. Schoenbaum G, Setlow B, Saddoris MP, Gallagher M. Encoding predicted outcome and acquired value in orbitofrontal cortex during cue sampling depends upon input from basolateral amygdala. Neuron. 2003;39:855-67.

6. Chau BK, Sallet J, Papageorgiou GK, Noonan MP, Bell AH, Walton ME, et al. Contrasting roles for orbitofrontal cortex and amygdala in credit assignment and learning in macaques. Neuron. 2015;87:1106-18.

\title{
Regulation of fear extinction and relapse by hippocampal
}

\section{engrams}

\author{
Michael R. Drew iD ${ }^{1}$ and Emma T. Brockway ${ }^{1}$ \\ Neuropsychopharmacology (2020) 45:228-229; https://doi.org/10.1038/s41386-019-0481-x
}

Maladaptive fears are often treated using therapies based on extinction-re-exposure to a feared stimulus in a safe environment. Although these treatments can be effective, relapse is common [1]. Since the time of Pavlov, relapse has been thought to indicate that extinction is new learning, not unlearning [2]. From this perspective, relapse is a mnemonic phenomenon in which two opposing memory traces vie for expression. Recent work suggests that the hippocampus is an arena in which this competition plays out.

Experiments in mice demonstrate that the hippocampal dentate gyrus (DG) generates a contextual fear "engram." Immediate-early gene-based tagging of neurons active during acquisition of contextual fear-fear of the chamber in which a footshock was given-shows that these neurons are reactivated during recall of contextual fear [3]. Furthermore, optogenetic experiments demonstrate that this reactivation is necessary and sufficient for expression of contextual fear [3, 4]. Two recent studies investigated what happens to hippocampal fear ensembles when contextual fear is extinguished.

The first of these studies, by Lacagnina et al. [5], used a transgenic mouse line to tag neurons active during acquisition or extinction of contextual fear. During a test session shortly after extinction training, fear acquisition neurons were suppressed and extinction neurons were reactivated. A month after extinction training mice displayed spontaneous recovery (relapse) of fear, and the pattern reversed: fear acquisition neurons were reactivated while extinction neurons were suppressed. The results suggest that the DG generates distinct fear acquisition and extinction representations, and competition between these representations determines whether fear is suppressed or recovers after extinction. Consistent with this interpretation, optogenetic manipulations demonstrated that reactivation of extinction neurons is necessary for suppression of fear after extinction, whereas reactivation of fear acquisition neurons is necessary for spontaneous recovery [5]

The other recent study, by Khalaf et al. [6], highlights that fear acquisition neurons also play an important role in extinction learning. In this study, neurons active during recall of a remote fear memory (acquired a month before extinction training) were tagged. Reactivation of these neurons was necessary for effective extinction, and artificial stimulation of these neurons improved extinction. For extinction to be effective, the fear acquisition memory must be reactivated during extinction training. Whether these findings apply to recent fear memories, like those studied in the Lacagnina et al. [5] experiments, is not yet known.

These studies reveal new ensemble mechanisms of fear extinction and relapse and raise some interesting questions. For instance, what aspects of extinction training stimulate creation of hippocampal extinction representations, and how do these representations suppress fear? Do they do so by activating extra-hippocampal fear-suppressive networks? In the Lacagnina study, why did fear acquisition neurons become more active over time and extinction neurons less active? Is it because of intrinsic differences between the two populations, changes in upstream input pathways, or different plasticity mechanisms involved in fear acquisition and extinction? Addressing these and other questions raised by the Lacagnina and Khalaf papers may provide keys to making extinction more resistant to relapse.

\section{FUNDING AND DISCLOSURE}

M.R.D. was supported by $\mathrm{NIH}$ grants R01 MH102595 and R01 $\mathrm{MH} 117426$. E.T.B. was supported by NIH grant T32MH106454. The authors declare no competing interests.

\section{ADDITIONAL INFORMATION}

Publisher's note: Springer Nature remains neutral with regard to jurisdictional claims in published maps and institutional affiliations.

\section{REFERENCES}

1. Vervliet B, Craske MG, Hermans D. Fear extinction and relapse: state of the art. Annu Rev Clin Psychol. 2013;9:215-48. 